\title{
Non-supersymmetric AdS and the swampland
}

\author{
Hirosi Ooguri And Cumrun VAFA
}

\begin{abstract}
We propose to sharpen the weak gravity conjecture by the statement that, except for BPS states in a supersymmetric theory, the gravitational force is strictly weaker than any electric force and provide a number of evidences for this statement. Our conjecture implies that any non-supersymmetric anti-de Sitter vacuum supported by fluxes must be unstable, as is the case for all known attempts at such holographic constructions.
\end{abstract}

\section{Introduction}

The weak gravity conjecture (WGC) [1] is an example of how seemingly consistent low energy gravitational theories can fail to have ultra-violet completion and instead belong to the swampland [2]; see [3] for more swampland constraints. Recently, there have been a number of papers checking and extending the WGC and applying it to cosmology [4,7, 9-12]. The conjecture has also been related to the holographic principle in [13. In this brief note, we propose sharpening of the WGC and point out that it implies that nonsupersymmetric anti-de Sitter (AdS) vacua supported by fluxes are unstable.

The WGC states that the mass of a particle carrying a basic unit of charge or its small multiple is less than or equal to that predicted if the particle were an extremal black hole. In other words, the gravitational force is equal or weaker than the electric force between such particles. The same idea applies to charged branes, where the tension for a basic brane charge or its small multiple should be less than or equal to that of the corresponding extremal black brane. The fact that it can sometimes be equal is well-known in string theory: BPS states in a supersymmetric theory saturate this relation. When the equality holds, the gravitational attraction and the electric repulsion of branes cancel out exactly.

In this paper we conjecture that the inequality between gravitational and electric forces is saturated if and only if the underlying theory is supersymmetric and the states under consideration are BPS with respect to the 
supersymmetry. We study a number of examples and provide evidences for this new conjecture.

This may seem like an innocuous extension of the WGC, but it has dramatic consequences. In particular, it implies that non-supersymmetric AdS vacua supported by fluxes ${ }^{1}$ must be unstable and that their effective theories belong to the swampland, even if they may look consistent. In all known top-down constructions from M/string theory, AdS geometry is supported by some flux. The sharpened version of the WGC predicts that there is a brane charged with respect to the flux and with tension less than the charge. As shown in [14] and reviewed in section 3 of this paper, such branes can nucleate in AdS, and the Coulomb repulsion by the flux wins over the tension to expand the brane. It then reaches the boundary of AdS within finite time, reducing the flux.

We can also understand this instability in the following way. To construct AdS holography in $\mathrm{M} /$ string theory, we typically start with a large number of extremal branes and take its near horizon limit. The sharpened version of the WGC implies that, unless the branes are BPS states of a supersymmetric theory, their electric repulsion wins over their gravitational attraction and this system is unstable. In the gravitational dual description, which will presumably exist when the extremal brane is large, the decay process can be slow and it can exist as a quasi-stable state. However, due to the gravitational time delay effect, the lifetime becomes shorter as we measure it closer to the horizon. In the near horizon limit, the decay becomes instantaneous. Therefore holographic duality may continue to exist for non-supersymmetric system, but its near horizon geometry, which leads to AdS dual description, does not exist for the non-supersymmetric system because of this instability.

The lack of non-supersymmetric AdS dual description, motivates us to propose our second conjecture, which is basically removing the assumption that the AdS is supported by the flux: We conjecture that nonsupersymmetric AdS holography is not realizable in a consistent quantum theory with low energy description in term of the Einstein gravity coupled to a finite number of matter fields, even if the AdS is not supported by a flux. We are restricting to theories with the Einstein gravity description in low energy since the WGC is motivated by black hole physics ${ }^{2}$ Note that this second conjecture does not follow from the sharpened version of the

\footnotetext{
${ }^{1} \mathrm{By}$ this we mean that there is a $(p+1)$-form gauge potential whose field strength along AdS does not vanish and is given by $F_{\mu_{1}, \ldots, \mu_{p+2}}=N \epsilon_{\mu_{1}, \ldots, \mu_{p+2}}^{\mathrm{AdS}}$.

${ }^{2}$ Therefore, topological field theories such as the Chern-Simons gauge theory and the Donaldson theory (though they have diffeomorphism invariance) are excluded from the scope of this conjecture.
} 
WGC and it is conceivable that the sharpened version of WGC is correct but this second conjecture is false.

Note that our second conjecture would have counter-examples if we relax the condition that the number of matter fields is finite. For example, nonsupersymmetric higher spin theories of the Vasiliev-type may be quantized consistently in AdS, and some of them may be dual to non-supersymmetric conformal field theories [15]. However, these theories contain infinite towers of higher spin fields. Another example is the Sachdev-Ye-Kitaev model [16 18. Although this model saturates the chaos bound on the Lyapunov exponent [18] and some of its correlation functions are well-approximated by the dilaton gravity in two dimensions first studied in [19, 20, its spectrum contains a tower of states with an approximately integer spacing, which can be interpreted as stringy states in the bulk whose tension is of the order of the AdS radius [21, 22]. Thus this theory is not a pure Einstein gravity coupled to a finite number of fields at the AdS scale.

The lack of explicit examples of non-supersymmetric AdS holography is often attributed to technical difficulties in estimating non-perturbative effects. Our conjecture suggests that there is a more fundamental reason for the lack of examples. If true, the absence of non-supersymmetric AdS vacua will have a significant implication on applications of the AdS/CFT correspondence to condensed matter physics, quantum information, hadron physics, and other areas in theoretical physics since interesting models in these contexts are typically those without supersymmetry.

Conformal field theory dual to the Einstein gravity with a finite number of matter fields must satisfy the gap condition: such a theory can contain only a small number of primary fields whose operator products generate all primary fields up to a certain energy scale, which can be made parametrically large in the large $N$ limit. The bulk reconstruction argument seems to suggest that the converse is also true [23, 24]. If we accept this premise, our conjecture would imply that non-supersymmetric conformal field theories cannot satisfy the gap condition.

Our conjectures can have highly non-trivial consequences. For example, our second conjecture, combined with the observation in [25] that the standard model with minimal Majorana neutrino masses compactified on a circle leads to non-supersymmetric $\mathrm{AdS}_{3}$ vacua with large radius ${ }^{3}$ rules out minimal Majorana neutrino masses for standard model to avoid this possibility!

\footnotetext{
${ }^{3} \mathrm{We}$ are assuming that there is no hidden mechanism in the standard model destablizing the solutions found in [25].
} 
The organization of this note is as follows. In section 2, we discuss several examples for the WGC and show how they satisfy the strict inequality for non-BPS states. In section 3 we motivate our conjecture that nonsupersymmetric AdS holography does not exist and discuss several examples to support the conjecture.

\section{Evidence for sharpened version of WGC}

In the simplest form, the WGC states that, for a $U(1)$ gauge theory coupled to gravity, there exists an elementary particle of mass $m$ and charge $Q$ satisfying the inequality,

$$
G m^{2}=\left(\frac{m}{M_{\mathrm{Pl}}}\right)^{2} \leq Q^{2} .
$$

Here $G$ is the Newton constant and $M_{\mathrm{Pl}}=1 / G$ is the Planck mass. The charge $Q$ is supposed to be the basic unit of the electric charge or a small multiple of it. We assume $m<M_{\mathrm{Pl}}$ so that any charged black hole with masses greater than $M_{\mathrm{Pl}}$ and satisfying the Reissner-Nordström bound is able to emit such a particle. We have suppressed a numerical factor that depends on spacetime dimensions; the precise form of the inequality is given later. The reason for allowing equality in the above is that this can be saturated in a supersymmetric theory with BPS states, which often happens in string theory. Otherwise, allowing the equality above seems unnatural for the following reasons.

One of the original motivations for the WGC in [1] was to allow extremal black holes to decay unless protected by supersymmetry since their existence would lead to a large number of remnants. If we interpret this requirement literarily, 2.1) should not include the equality since extremal black holes can only emit particles strictly below the Reissner-Nordström bound. Particles at the bound would have vanishing phase space for their emissions. Moreover, such particles would be on the verge of violating the WGC! If we allow the equality, there should be a good reason why small perturbation to the theory would not tip the balance in a wrong direction and violate the WGC. In the supersymmetric case, the robustness is guaranteed by the BPS condition. For a non-supersymmetric theory, however, if the inequality is saturated, there is no known mechanism to keep this balance from tipping in the wrong direction. This motivates the stronger conjecture:

The $W G C$ is saturated if and only if the theory is supersymmetric and the state in question is a BPS state. 
The same sharpening of the conjecture can be considered for branes also: a consistent gravity theory with a $(p+1)$-form gauge field must contain $p$ branes below the $p$-brane version of the Reissner-Nordström bound, unless the $(p+1)$-form is in the supergravity multiplet. Such $p$-branes would ensure that black $p$-branes are unstable unless protected by supersymmetry.

Note that the original paper [1] on the WGC does not claim that there is a particle with $|m / Q| \leq M_{\mathrm{Pl}}$ for every possible charge $Q$. In fact, the paper described counter-examples to it. The sharpened version of the conjecture also applies only to particles with minimal $|m / Q|$ and the statement is that there are some non-supersymmetric states for which this ratio is strictly less than $M_{\mathrm{Pl}}$. The lowest $|m / Q|$ ratio may occur for a state whose charge is a small multiple of the basic charge.

Let us present evidences to demonstrate our conjecture. Suppose there is a family of stable non-BPS states which carry different values of $Q$ and become extremal black holes in the limit of large $Q$, namely, $m(Q) /|Q| \rightarrow$ $M_{\mathrm{Pl}}$ for $Q \rightarrow \infty$. In [28, finite $Q$ corrections to $m(Q) /|Q|$ are computed for non-supersymmetric black holes using higher derivative terms in the low energy effective action derived from the heterotic string theory compactified on a torus. It turns out that leading corrections take the form,

$$
\frac{m(Q)}{M_{\mathrm{Pl}}}=|Q|(1-\varepsilon(Q)),
$$

where $\varepsilon(Q)$ is always positive. This shows that, as the charge $Q$ decreases, the extremal black hole becomes super-extremal due to higher derivative corrections and the strict WGC inequality is satisfied.

There are several examples of stable non-supersymmetric states that satisfy the strict inequality. One class of such states has already been discussed in the original paper [1]. Consider the Narain compactification of the heterotic string theory on $T^{d}$. The low energy theory has $U(1)^{16+2 d}$ gauge symmetry, and their charges make an even self-dual lattice,

$$
\left(p_{L}, p_{R}\right) \in \Gamma^{16+d, d} .
$$

In our convention, the left-mover is bosonic and the right-mover is supersymmetric. Let us look for the lowest mass state for a given set of charges. Recall that the mass squared of a perturbative string state is given by,

$$
m^{2}=\frac{1}{2} p_{L}^{2}+N_{L}-1=\frac{1}{2} p_{R}^{2}+N_{R},
$$


where $N_{L}$ and $N_{R}$ are amounts of excitations in the left and right-movers. To minimize the mass, we should try to take $N_{L}$ and $N_{R}$ as small as possible. Since $\frac{1}{2}\left(p_{L}^{2}-p_{R}^{2}\right)=N_{R}-N_{L}+1$, we can set both $N_{L}$ and $N_{R}$ to be zero only if $p_{L}^{2}-p_{R}^{2}=2$. If $p_{L}^{2}-p_{R}^{2}<2$, we can set $N_{R}=0$, but $N_{L}$ has to be non-zero. The corresponding state is BPS and known as a Dabholkar-Harvey state [26, 27]. In that case, $m^{2}=\frac{1}{2} p_{R}^{2}$, saturating the WGC bound. On the other hand, if $p_{L}^{2}-p_{R}^{2}>2$, we can choose $N_{L}=0$ but $N_{R}$ has to be non-zero. The resulting state is not BPS, and we have,

$$
m^{2}=\frac{1}{2} p_{L}^{2}-1
$$

leading to the strict inequality $m^{2}<\frac{1}{2} p_{L}^{2}$ because of the Casimir energy $(-1)$ for the bosonic left-moving sector on the worldsheet. We thus see the clear correlation between breaking supersymmetry and realizing the strict WGC bound in this example. For large $p_{L}^{2}$, the above mass formula takes the form of 2.2 with $\varepsilon \sim 1 / p_{L}^{2}$, which is positive and vanishes in the large charge limit $p_{L}^{2} \rightarrow \infty$ as expected from the analysis of [28].

The above example is in the weak coupling limit of the heterotic string theory. Both the WGC and its sharpened version are supposed to hold for arbitrary coupling constant. We can test the sharpened version in the strong coupling limit of the heterotic string theory compactified on $S^{1}$ by using its duality to the Type I string theory [29, 30]. The low energy dynamics of the Type I D-string with the winding number $n$ on $S^{1}$ can be described by an $O(n)$ gauge theory on the D-string worldsheet. In particular, when $n=1$, the worldsheet theory on the D-string is identical to that on the fundamental heterotic string on $S^{1}$, except that the tension and the radius of $S^{1}$ are different and relatd to those on the heterotic string side by the duality rules. For example, the $O(1)=\mathbb{Z}_{2}$ gauge symmetry on the D-string worldsheet acts as the GSO projection on the heterotic string side. The spectrum on the singly-wound D-string therefore has the same structure as that on the heterotic string worldsheet discussed in the above, and nonBPS excitations of the D-string satisfy the strict WGC inequality. Thus, the sharpened version of the WGC works for both weak and strong coupling constants of heterotic string in this case. It would be interesting to generalize this analysis for $n>1$.

Our conjecture also leads to a number of mathematically falsifiable predictions. Consider $\mathrm{M}$ theory on K3, which is dual to the heterotic string on $T^{3}$. Since the large volume limit of K3 in M theory corresponds to the strong coupling limit in the heterotic string, K3 geometry can be used to probe the regime that is not accessible by the heterotic string perturbation. This is 
specially so for non-supersymmetric states, whose masses are not protected as we change the coupling constant. Can non-supersymmetric states have masses less than their charge in the large volume limit of $\mathrm{K} 3$ on the Mtheory side? Consider $\mathrm{H}_{2}(\mathrm{~K} 3, \mathbf{Z})$. This gives a lattice with inner product, obtained by intersection of a pair of 2-cycles. It is known that this lattice has signature $(19,3)$. Moreover the metric on $K 3$ induces a projection on 2 -forms to self-dual and anti-self-dual forms. This is called a polarization on $H^{2}(K 3, \mathbf{Z})$ which induces a polarization on the dual space $H_{2}(K 3, \mathbf{Z})$. Using this we can identify $H_{2}(K 3, \mathbf{Z})$ with the corresponding lattice of momenta $\left(p_{L}, p_{R}\right)$ for heterotic strings on $T^{3}$. When $p_{L}^{2}-p_{R}^{2} \leq 2$, the 2-cycle class can be realized as a holomorphic curve of genus $g$, where

$$
p_{L}^{2}-p_{R}^{2}=2-2 g .
$$

M2 branes wrapping holomorphic curves lead to BPS states, and they are dual to the heterotic string BPS states. On the other hand, if $p_{L}^{2}-p_{R}^{2}>$ 2 , there is no holomorphic curve. We can compare the minimum mass of the M2 brane wrapping such a 2-cycle to its charges. In the large volume limit the mass of such an M2 brane is equal to the area of the 2-cycle it wraps. In particular suppose we have a polarization such that a charge vector associated to a 2-cycle is of the form $\left(p_{L}, 0\right)$ with $p_{L}^{2}>2$. Then this class cannot be represented by a holomorphic cycle. On the other hand, suppose we have a minimal surface in this class. Let $A$ denote the area of this cycle (where the volume of $K 3$ is normalized so that for supersymmetric cycles $\left.A=\left|p_{R}\right|\right)$. Then the WGC implies

$$
A<\left|p_{L}\right|
$$

which would be interesting to verify.

There are other predictions that follow from the sharpened version of the WGC. For example, consider $\mathcal{N}=1$ supersymmetric theories in four dimensions coupled to supergravity. If the theory has $U(1)$ gauge symmetries, the sharpened version of WGC predicts that there is a charged state with mass strictly less than charge for small multiples of basic $U(1)$ charge. This is because there are no BPS states in $\mathcal{N}=1$ theories in four dimensions. For example, consider $\mathrm{M}$ theory compactified on a $G_{2}$ holonomy manifold, leading to $\mathcal{N}=1$ supersymmetric theory in four dimensions. For each 2 cycle in the manifold, we get a $U(1)$ gauge factor. M2 branes wrapping the corresponding 2-cycles gives charged states. The sharpened version of the WGC predicts that the mass of the corresponding M2 brane is less than the corresponding charge. 
Our final evidence is provided by the behavior of the WGC inequality under dimensional reduction. In [31, the precise form of the inequality is determined for a charged particle with mass $m$ and charge $Q$ coupled to the Einstein-Maxwell-dilaton system in $d$ dimensions as,

$$
8 \pi G\left(\frac{\alpha^{2}}{2}+\frac{d-3}{d-2}\right) m^{2} \leq Q^{2}
$$

where $\alpha$ is the dilaton coupling to the Maxwell term as $e^{-\alpha \phi} F^{2}$ in the Einstein frame for the metric. If the dilaton is massless, it affects long range features of black hole solutions and contributes to the WGC inequality. Since the factor $(d-3) /(d-2)$ is an increasing function of $d$, if the dilaton coupling $\alpha$ is independent of $d$, a particle saturating the WGC inequality in $d$ dimensions would obey the strict WGC inequality under toroidal compactification to lower dimensions. However, the value of $\alpha$ can change under compactification since additional massless scalar fields such as the radion for the size of the internal torus can be generated. They can mix with the dilaton in the original theory and modify the effective value of $\alpha$. Remarkably, it was shown in 31] that, if compactification preserves supersymmetry, the value of $\alpha$ is modified exactly in such a way to compensate for the $d$ dependence in $(d-3) /(d-2)$ and to make the WGC inequality (2.3) invariant under dimensional reduction. With supersymmetry, the dilaton and the radion remain strictly massless in infrared. If supersymmetry is broken, scalar fields generically become massive and cease to contribute to long range features of black hole solutions. In such a case, $\alpha$ cannot compensate for the $d$ dependence in in 2.1, and the inequality cannot be saturated after dimensional reduction if it holds in the original theory. This gives yet another evidence for the sharpened version of the WGC, i.e., the inequality cannot be saturated without supersymmetry.

\section{Gravitational instability of non-supersymmetric AdS}

The sharpened version of the WGC implies that any non-supersymmetric AdS geometry supported by flux is unstable in a consistent quantum theory of gravity with low energy description in terms of the Einstein gravity coupled to a finite number of matter fields. Equivalently, there are no nonsupersymmetric conformal field theories whose holographic dual have such gravity description. These statements follow from our sharpened version of the WGC combined with the analysis in [14] that $\mathrm{AdS}_{p+2}$ geometry supported by a $(p+2)$ form flux is unstable if there are $p$-branes charged with 
respect to the flux and whose charges are less than their tensions. In the Euclidean $\mathrm{AdS}_{p+2}$ geometry,

$$
d s^{2}=\cosh ^{2} \rho d \tau^{2}+d \rho^{2}+\sinh ^{2} \rho d \Omega_{p}^{2},
$$

a spherical $p$-brane on $S^{p}$ at radius $\rho(\tau)$ evolving in the Euclidean time $\tau$ has the action proportional to,

$$
S_{E} \sim \int\left(\sinh ^{p} \rho \sqrt{\cosh ^{2} \rho+(d \rho / d \tau)^{2}}-q \cdot \sinh ^{p+1} \rho\right) d \tau,
$$

where $q$ is the charge/tension ratio normalized in such a way that the Reissner-Nordström bound is at $q=1$. It was shown in [14 that, when $q>1$, there is an instanton solution which nucleates a spherical brane with radiu $\rho_{\mathrm{n}}$ given by $\tanh \rho_{\mathrm{n}}=1 / q$. The brane then expands in the Lorentzian AdS as $\cosh \rho=\cosh \rho_{\mathrm{n}} / \cos t$ and reaches the AdS boundary at $\rho=\infty$ in finite time of $t=\pi / 2$, reducing the flux of the geometry.

Unlike flat or de Sitter vacua, the instability of AdS vacua can be detected instantaneously in their would-be dual conformal field theories [32, 33. This is because any observer in AdS gets access to the entire Cauchy surface within a finite amount of time. In particular, an observer at the boundary of AdS can get access to an infinite volume space near the boundary within an infinitesimal amount of time. Since the instability process discussed in the above causes a finite decay probability per unit volume of AdS, the decay would happen instantaneously when seen from the boundary. Therefore, the dual conformal field theory cannot exist even as a meta-stable state.

The instantaneous nature of the decay can also be explained as follows. Consider an extremal $p$-brane, whose near horizon reproduces the $\mathrm{AdS}_{p+2}$ geometry in the above. According to the sharpened version of the WGC, if the extremal $p$-brane is not protected by supersymmetry, there is a superextremal brane charged with respect to the $(p+2)$-form flux and with tension less than its charge. The extremal brane can decay by emitting such super-extremal branes. A large extremal brane can exist as a quasi-stable state since its decay rate can be small. However, the rate becomes larger as we measure it closer to the horizon. In the near horizon limit, the decay becomes instantaneous, consistently with the conclusion of the previous paragraph.

There have been several proposals for non-supersymmetric AdS vacua. One can start with $\operatorname{AdS}_{5} \times S^{5}$, which is dual to the $\mathcal{N}=4$ supersymmetric 
Yang-Mills theory in four dimensions, and divide the $S^{5}$ by a discrete subgroup $\Gamma$ of its $S U(4)$ rotational symmetry 34 . Supersymmetry is completely broken if the orbifold group $\Gamma$ does not fit within an $S U(3)$ subgroup of the $S U(4)$ symmetry. It turns out that its gauge theory is typically not conformal when $\Gamma$ breaks supersymmetry completely since couplings for double-trace operators cannot be stabilized under the renormalization group flow. It was shown in [35, 36] that, when the action of $\Gamma$ on $S^{5}$ has a fixed point or when $\Gamma$ acts freely but the ' $t$ Hooft coupling is small, a double-trace operator fails to find a fixed point. In this case, a closed string tachyon emerges in a twisted sector, and the $\mathrm{AdS}_{5} \times S^{5} / \Gamma$ geometry becomes perturbatively unstable.

When $\Gamma$ acts freely on $S^{5}$ and the 't Hooft coupling is large, there is no tachyon in the spectrum. However, the geometry is still unstable nonperturbatively. If $\Gamma$ acts freely on $S^{5}$, there are non-contractible cycles on $S^{5} / \Gamma$. It was shown in 32 that there is an instanton solution which describe a tunneling of the $\operatorname{AdS}_{5} \times S^{5} / \Gamma$ geometry into nothing. In the instanton geometry, one of the supersymmetry breaking cycle shrinks to a point and the geometry terminates over an $S^{4}$ homologous to the $S^{4}$ at the infinity of $\mathrm{AdS}_{5}$. This is similar to the instanton used in [37] to demonstrate the instability of the Kaluza-Klein vacuum. Here the role of the Kaluza-Klein circle is played by the supersymmetry breaking cycle in $S^{5} / \Gamma$. To conserve the 5-form flux in the geometry, an appropriate number of D3 branes must wrap the $S^{4}$. After analytical continuation to the Lorentzian signature, the $S^{4}$ becomes an expanding bubble of $S^{3}$, which grows and reaches the boundary of $\mathrm{AdS}_{5}$ in finite time, consuming the entire spacetime in the process. The expanding bubble wrapped by D3 branes can be regarded an example of branes predicted by the weak gravity conjecture, except that its D3 charge is large. In this case, the brane carries exactly the amount of charge that cancels the 5 -form flux on $S^{5} / \Gamma$, rather than the minimum D3 brane charge.

There have been other proposals for non-supersymmetric AdS vacua, but none of them has been demonstrated to be stable. The non-supersymmetric $\mathrm{AdS}_{4}$ vacua in the massive IIA supergravity discussed in [38] allow marginal decays in their approximation, and the magnetic AdS geometry discussed in [39] does not stabilize the dilaton.

Since we know that there are conformal field theories without supersymmetry, it is reasonable to ask: Why can't they have holographic AdS duals? To understand this, let us consider one more example where one attempts to construct a holographic dual to a non-supersymmetric conformal field theory. Consider the D1-D5 string on K3, whose low energy theory is described by the two-dimensional supersymmetric sigma-model with the symmetric product of K3's as its target space. It is dual to the Type IIB string theory 
on $\mathrm{AdS}_{3} \times S^{3} \times \mathrm{K} 3$. However, the gravity approximation is not valid at the orbifold limit of the symmetric product target space since the D1-D5 system there has more low energy states than predicted by the supergravity [40-43]. We have to deform the sigma model away from the orbifold point by marginal operator corresponding to blow up modes where a pair of K3 approach one another. This is needed in order to land in a regime where gravity dual does not have infinitely many light modes. This shows that the existence of marginal operators of the symmetric product of the orbifold theory plays a key role in realizing the holographic dual described by the Einstein gravity with a finite number of matter fields. Now, let us contrast this with its non-supersymmetric counterpart. The bosonic sigma model on the symmetric product of K3's is conformal at least in the orbifold limit. We can ask if it has a holographic dual. In the supersymmetric case, we saw that the existence of holographic dual is crucially hinged on the exactly marginal operators which can take the theory away from the orbifold limit. In the non-supersymmetric case, it is easy to check that the requisite blow-up mode is not marginal and that we cannot go away from the orbifold point. This is consistent with our conjecture that this theory will fail to have a holographic dual as a weakly coupled gravity.

\section{Acknowledgments}

We would like to thank N. Arkani-Hamed, T. Dumitrescu, D. Harlow, I. Klebanov, J. Maldacena, G. Remmen, T. Rudelius, A. Sen, S. Shenker, A. Strominger, and E. Witten for discussions. The research of HO is supported in part by U.S. Department of Energy grant DE-SC0011632, by the Simons Investigator Award, by the World Premier International Research Center Initiative, MEXT, Japan, by JSPS Grant-in-Aid for Scientific Research C26400240, and by JSPS Grant-in-Aid for Scientific Research on Innovative Areas 15H05895. HO also thanks the hospitality of the Aspen Center for Physics, which is supported by the National Science Foundation grant PHY1066293, and of the the Center for Mathematical Sciences and Applications and the Center for the Fundamental Laws of Nature at Harvard University. The research of CV is supported by the NSF grant PHY-1067976.

\section{References}

[1] N. Arkani-Hamed, L. Motl, A. Nicolis, and C. Vafa, The string landscape, black holes and gravity as the weakest force, JHEP 0706 (2007) 060. arXiv: hep-th/0601001. 
[2] C. Vafa, The string landscape and the swampland, arXiv:hep-th/ 0509212.

[3] H. Ooguri and C. Vafa, On the geometry of the string landscape and the swampland, Nucl. Phys. B 766 (2007) 21. arXiv:hep-th/0605264.

[4] A. de la Fuente, P. Saraswat, and R. Sundrum, Natural inflation and quantum gravity, Phys. Rev. Lett. 114 (2015), no. 15, 151303. arXiv: 1412.3457 [hep-th].

[5] T. Rudelius, Constraints on axion inflation from the weak gravity conjecture, JCAP 1509 (2015) 020. arXiv:1503.00795 [hep-th].

[6] J. Brown, W. Cottrell, G. Shiu, and P. Soler, Fencing in the swampland: quantum gravity constraints on large field inflation, JHEP 1510 (2015) 023. arXiv: 1503.04783 [hep-th].

[7] T. C. Bachlechner, C. Long, and L. McAllister, Planckian axions and the weak gravity conjecture, JHEP 1601 (2016) 091. arXiv:1503.07853 [hep-th].

[8] C. Cheung and G. N. Remmen, Naturalness and the weak gravity conjecture, Phys. Rev. Lett. 113 (2014), 051601. DOI:10.1103/ PhysRevLett.113.051601; arXiv:1402.2287 [hep-ph].

[9] B. Heidenreich, M. Reece, and T. Rudelius, Weak gravity strongly constrains large-field axion inflation, JHEP 1512 (2015) 108. arXiv: 1506.03447 [hep-th].

[10] L. E. Ibanez, M. Montero, A. Uranga, and I. Valenzuela, Relaxion monodromy and the weak gravity conjecture, JHEP 1604 (2016) 020. arXiv: 1512.00025 [hep-th].

[11] A. Hebecker, F. Rompineve, and A. Westphal, Axion monodromy and the weak gravity conjecture, JHEP 1604 (2016) 157. arXiv: 1512.03768 [hep-th].

[12] A. Uranga and G. Shiu, Lectures at the 10th Asian Winter School on Strings, Particles and Cosmology held at OIST, Okinawa, Japan. The lecture notes are available at http://groups.oist.jp/aws/ lecturers.

[13] D. Harlow, Wormholes, emergent gauge fields, and the weak gravity conjecture, JHEP 1601 (2016) 122. arXiv:1510.07911 [hep-th]. 
[14] J. M. Maldacena, J. Michelson, and A. Strominger, Anti-de Sitter fragmentation, JHEP 9902 (1999) 011. arXiv:hep-th/9812073.

[15] See for example, S. Giombi, TASI lectures on the higher spin - CFT duality, arXiv:1607.02967 [hep-th], and references therein.

[16] S. Sachdev and J.-W. Ye, Gapless spin fluid ground state in a random, quantum Heisenberg magnet, Phys. Rev. Lett. 70 (1993) 3339. arXiv: cond-mat/9212030.

[17] S. Sachdev, Holographic metals and the fractionalized Fermi liquid, Phys. Rev. Lett. 105 (2010) 151602. arXiv:1006.3794 [hep-th].

[18] A. Kitaev, A simple model of quantum holography, KITP strings seminar and Entanglement 2015 program (given on February 15, April 7, and May 27, 2015),

[19] R. Jackiw, Lower dimensional gravity, Nucl. Phys. B 252 (1985) 343.

[20] C. Teitelboim, Gravitation and Hamiltonian structure in two space-time dimensions, Phys. Lett. B 126 (1983) 41.

[21] J. Polchinski and V. Rosenhaus, The spectrum in the Sachdev-Ye-Kitaev model, JHEP 1604 (2016) 001. arXiv:1601.06768 [hep-th].

[22] J. Maldacena and D. Stanford, Comments on the Sachdev-Ye-Kitaev model, arXiv:1604.07818 [hep-th].

[23] I. Heemskerk, J. Penedones, J. Polchinski, and J. Sully, Holography from conformal field theory, JHEP 0910 (2009) 079. arXiv:0907.0151 [hep-th].

[24] I. Heemskerk, D. Marolf, J. Polchinski, and J. Sully, Bulk and transhorizon measurements in AdS/CFT, JHEP 1210 (2012) 165. arXiv: 1201.3664 [hep-th].

[25] N. Arkani-Hamed, S. Dubovsky, A. Nicolis, and G. Villadoro, Quantum horizons of the standard model landscape, JHEP 0706 (2007) 078. arXiv:hep-th/0703067.

[26] A. Dabholkar and J. A. Harvey, Nonrenormalization of the superstring tension, Phys. Rev. Lett. 63 (1989) 478.

[27] A. Dabholkar, G. W. Gibbons, J. A. Harvey, and F. Ruiz Ruiz, Superstrings and solitons, Nucl. Phys. B 340 (1990) 33. 
[28] Y. Kats, L. Motl and M. Padi, Higher-order corrections to masscharge relation of extremal black holes, JHEP 0712 (2007) 068. arXiv: hep-th/0606100.

[29] E. Witten, String theory dynamics in various dimensions, Nucl. Phys. B 443 (1995) 85. arXiv:hep-th/9503124.

[30] J. Polchinski and E. Witten, Evidence for heterotic - type I string duality, Nucl. Phys. B 460 (1996) 525. arXiv:hep-th/9510169.

[31] B. Heidenreich, M. Reece and T. Rudelius, Sharpening the weak gravity conjecture with dimensional reduction, JHEP 1602 (2016) 140. arXiv:1509.06374 [hep-th].

[32] G. T. Horowitz, J. Orgera, and J. Polchinski, Nonperturbative instability of $A d S_{5} \times S^{5} / \mathbb{Z}_{k}$, Phys. Rev. D 77 (2008) 024004. arXiv:0709.4262 [hep-th].

[33] D. Harlow, Metastability in anti de Sitter space, arXiv:1003.5909 [hep-th].

[34] S. Kachru and E. Silverstein, 4-D conformal theories and strings on orbifolds, Phys. Rev. Lett. 80 (1998) 4855. arXiv: hep-th/9802183.

[35] A. Dymarsky, I. R. Klebanov, and R. Roiban, Perturbative search for fixed lines in large $N$ gauge theories, JHEP 0508 (2005) 011. arXiv:hep-th/0505099.

[36] A. Dymarsky, I. R. Klebanov, and R. Roiban, Perturbative gauge theory and closed string tachyons, JHEP 0511 (2005) 038. arXiv: hep-th/0509132.

[37] E. Witten, Instability of the Kaluza-Klein vacuum, Nucl. Phys. B 195 (1982) 481.

[38] P. Narayan and S. P. Trivedi, On the stability of non-supersymmetric AdS vacua, JHEP 1007 (2010) 089. arXiv:1002.4498 [hep-th].

[39] A. Almuhairi and J. Polchinski, Magnetic $A d S_{2} \times \mathbb{R}^{2}$ : supersymmetry and stability, arXiv:1108.1213 [hep-th].

[40] R. Dijkgraaf, G. W. Moore, E. P. Verlinde, and H. L. Verlinde, Elliptic genera of symmetric products and second quantized strings, Commun. Math. Phys. 185 (1997) 197. arXiv:hep-th/9608096.

[41] J. de Boer, Large $N$ elliptic genus and AdS/CFT correspondence, JHEP 9905 (1999) 017. arXiv:hep-th/9812240. 
[42] C. A. Keller, Phase transitions in symmetric orbifold CFTs and universality, JHEP 1103 (2011) 114. arXiv:1101.4937 [hep-th].

[43] T. Hartman, C. A. Keller and B. Stoica, Universal spectrum of $2 d$ conformal field theory in the large c limit, JHEP 1409 (2014) 118. arXiv: 1405.5137 [hep-th].

Walter Burke Institute for Theoretical Physics

California Institute of Technology

PASADENA, CA 91125, USA

and Kavli Institute for the Physics and Mathematics

UNIVERSE UNIVERSITY OF TOKYO

KASHIWA, 277-8583, JAPAN

E-mail address: ooguri@caltech.edu

Center for the Fundamental Laws of Nature

Harvard University, Cambridge, MA 02138, USA

E-mail address: vafa@physics.harvard.edu 
